\title{
miR-21-5p targets PDHA1 to regulate glycolysis and cancer progression in gastric cancer
}

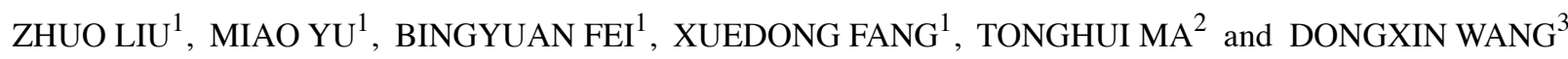 \\ ${ }^{1}$ Department of Gastrointestinal Colorectal and Anal Surgery, China-Japan Union Hospital of Jilin University, \\ Changchun, Jilin 130021; ${ }^{2}$ College of Basic Medical Sciences, Dalian Medical University, Dalian, \\ Liaoning 116044; ${ }^{3}$ Jilin Cancer Hospital, Changchun, Jilin 130021, P.R. China
}

Received December 15, 2017; Accepted July 13, 2018

DOI: $10.3892 / o r .2018 .6695$

\begin{abstract}
Pyruvate dehydrogenase A1 (PDHA1) is a component of the pyruvate dehydrogenase enzyme complex, which links glycolysis and the tricarboxylic acid cycle, and is important for cancer metabolism shift. PDHA1 downregulation has been revealed in several types of cancer to enhance glycolysis. However, the role of PDHA1 in gastric cancer remains largely unknown. In the present study, we found that PDHA1 was significantly downregulated in gastric cancer, and associated with poor prognosis. PDHA1 downregulation promoted gastric cancer glycolysis and cancer progression. miR-21-5p directly targeted PDHA1 to suppress PDHA1 expression, and promote glycolysis as well as cell proliferation in gastric cancer. Moreover, miR-21-5p was significantly upregulated in gastric cancer and negatively associated with PDHA1 expression in gastric cancer samples. Our results indicated that miR-21-5p targeted PDHA1 to regulate a metabolic switch and cancer progression in gastric cancer, and reveal the potential role of the miR-21-5p/PDHA1 axis in gastric cancer treatment.
\end{abstract}

\section{Introduction}

Gastric cancer is the third most lethal malignancy worldwide (1). Currently, the main option for treating gastric cancer is surgical resection combined with chemotherapy and/or radiotherapy. In recent years, due to the fast development of enterescopy and surgical techniques, the 5-year mortality for early gastric cancer has been significantly reduced. However, most patients with gastric cancer are diagnosed at an advanced

Correspondence to: Dr Dongxin Wang, Jilin Cancer Hospital, 1018 Huguang Road, Changchun, Jilin 130021, P.R. China

E-mail: dongxinwjl@163.com

Dr Tonghui Ma, College of Basic Medical Sciences, Dalian Medical University, 9 West Section Lvshun South Road, Dalian, Liaoning 116044, P.R. China

E-mail: tonghuim@126.com

Key words: microRNA, microRNA-21-5p, gastric cancer, metabolism, pyruvate dehydrogenase A1 stage and miss the optimal timing for surgical intervention. Even when curative resection is performed successfully, $\sim 40-65 \%$ of patients may still experience a recurrence of the disease. Therefore the 5-year mortality still remains high (2). Although extensive studies have been carried out to explore the possible mechanisms that underlie the development of gastric cancer, the exact mechanism remains ambiguous. Better understanding of the related mechanisms is important in order to establish new efficient targets and to develop novel treatments to manage gastric cancer.

Dysregulated metabolism frequently occurs in various cancer cells, and is considered as one of the important hallmarks of cancer (3). The famous 'Warburg effect' states that glucose consumption becomes a priority for cancer cells even in the presence of oxygen $(4,5)$. Blocking cancer glycolysis is considered to be a promising therapeutic strategy for cancer treatment. Pyruvate links glycolysis and the TCA cycle, thus enzymes involved in the pyruvate reaction play important roles in the metabolic node (6). Pyruvate dehydrogenase $\alpha 1$ (PDHA1) is a key component of the pyruvate dehydrogenase complex which catalyzes pyruvate decarboxylation and serves as a gate-keeper enzyme link between glycolysis and the mitochondrial citric acid cycle. The inhibition of pyruvate dehydrogenase in cancer cells increases the Warburg effect in cancer cells and renders cancer cells more malignant $(7,8)$. PDHA1 dysregulation has also been revealed to be associated with metabolic reprogramming in cancer cells $(9,10)$. It has been reported that decreased expression of PDHA1 predicted an unfavorable prognosis in ovarian carcinoma (11), and low PDHA1 protein expression may indicate the aggressive features of clear cell carcinoma (12). However, in gastric cancer, the role of PDHA1 has not been studied.

MicroRNAs (miRNAs) are one of the main reasons for protein level dysregulation. miRNAs are a class of small non-coding RNAs that are generally 19-25 nucleotides in length $(13,14)$. miRNAs regulate gene expression by binding to the 3'untranslated region (3'UTR) of the target mRNA, leading to inhibition of protein translation or cleavage of the target mRNA $(15,16)$. Accumulating evidence has revealed that miRNAs are involved in various biological process, including development, apoptosis and differentiation (17-20). Research has also revealed that dysregulation of miRNAs is associated with many diseases, including tumors (21). 
In the present study, we found that PDHA1 was downregulated in gastric cancer, and PDHA1 downregulation promoted glycolysis and gastric cancer cell progression. PDHA1 was directly regulated by $\mathrm{miR}-21-5 \mathrm{p}$. In addition, miR-21-5p was upregulated in gastric cancer, and promoted gastric cancer progression by regulating PDHA1. Our results indicated the role of the miR-21-5p/PDHA1 axis in regulating gastric cancer metabolism and progression, and suggests their potential value in gastric cancer treatment.

\section{Materials and methods}

Clinical specimens. Gastric cancer and matched adjacent normal tissues were collected from gastric cancer patients at China-Japan Union Hospital of Jilin University. The tissue samples from 46 patients ( 31 males and 15 females with age ranging from 43 to 71 years) were collected from November 2016 to September 2017. All carcinoma samples were obtained during surgery, snap-frozen in liquid nitrogen and stored at $-80^{\circ} \mathrm{C}$ until further analysis. In the present study, all the cases of gastric tumors were divided into low-grade (WHO I and II) or high-grade (WHO III and IV) for statistical analysis. This study was approved by the Ethics Committee of China-Japan Union Hospital of Jilin University. Written informed consent was obtained from all the patients or their guardians in accordance with the ethical committee standards.

RNA extraction and real-time quantitative polymerase chain reaction. Total RNA was extracted using TRIzol reagent (Invitrogen; Thermo Fisher Scientific, Inc., Waltham, MA, USA). Total RNA (500 ng) was used for cDNA synthesis using a High Capacity RNA-to-cDNA Kit (Applied Biosystems; Thermo Fisher Scientific, Inc.). miScript Reverse Transcription kit (Qiagen GmbH, Hilden, Germany) was used for reverse transcription of miRNAs. Quantitative real-time polymerase chain reaction (PCR) was carried out on an ABI PRISM 7500 Sequence Detection System (Applied Biosystems, Foster City, CA, USA) using SYBR Premix Ex Taq (Takara Biotechnology Co., Ltd., Dalian, China) according to the manufacturer's instructions. The miRNA sequence-specific reverse transcription (RT)-PCR for miR-21-5p and endogenous control U6 was performed using Hairpin- $\mathrm{it}^{\mathrm{TM}}$ miRNAs qPCR quantitation kit and U6 snRNA real-time PCR normalization kit (Shanghai GenePharma Co., Ltd., Shanghai, China). The primers for PDHA1 are: forward, 5'-GAGCTGAGCAGCTGTGTAAC-3' and reverse, 5'-TGCCAATCGTTACAGGTATTACAG-3'. The thermocycling conditions for miR-21-5p were: $94^{\circ} \mathrm{C}$ for $3 \mathrm{~min}$ (hold), 40 cycles at $94^{\circ} \mathrm{C}$ for $15 \mathrm{sec}$ (denaturation); $55^{\circ} \mathrm{C}$ for $25 \mathrm{sec}$ (annealing); $72^{\circ} \mathrm{C}$ for $25 \mathrm{sec}$ (elongation). The thermocycling conditions for PDHA1 were: $95^{\circ} \mathrm{C}$ for $20 \mathrm{sec}$ (enzyme activation), 40 cycles at $95^{\circ} \mathrm{C}$, for $3 \mathrm{sec}$ (denaturation); $60^{\circ} \mathrm{C}$ for $30 \mathrm{sec}$ (annealing/elongation). All reactions were performed in triplicate.

Immunohistochemistry and in situ hybridization. Tumor or matched normal tissues were fixed with $4 \%$ paraformaldehyde, and then the samples were embedded in paraffin and sectioned. Following overnight incubation at $4^{\circ} \mathrm{C}$ with the PDHA1 antibody (1:100; cat. no. 3205; Cell Signaling Technology, Inc., Danvers, MA, USA), the bound antibodies were detected with the biotin-streptavidin-peroxidase system (Vector Laboratories, Inc., Burlingame, CA, USA) using diaminobenzidine (Sigma-Aldrich; Merck KGaA, Darmstadt, Germany) as chromogen.

For the in situ hybridization assay, DIG-labeled locked nucleic acid (LNA)-based probe specific for miR-21-5p (Exiqon A/S, Vedbaek, Denmark) was introduced. In addition, the in situ hybridization signal was detected by overnight incubation at $4^{\circ} \mathrm{C}$ with horseradish peroxidase (HRP)-conjugated anti-digoxigenin 21H8 (1:200; cat. no. ab420; Abcam, Cambridge, MA, USA).

Transfection. The miR-21-5p mimics, mimics negative control, inhibitor, inhibitor negative control (inhibitor control), the small interfering RNA targeting PDHA1 (siPDHA1) and control-siRNA (siCon) were purchased from Shanghai GenePharma Co., Ltd. Cells were seeded at a density of $2-3 \times 10^{5}$ cells/well in 6 -well plates for $24 \mathrm{~h}$. The cells were then transfected with oligonucleotides or plasmid, using Lipofectamine 2000 (Invitrogen; Thermo Fisher Scientific, Inc.) following the manufacturer's instructions.

Construction and preparation of the PDHAl lentivirus. PDHA1 cDNA was amplified from an adult gastric tissue cDNA library. The recombinant lentiviruses were packaged using the pMagic 4.0 lentivirus pLV expression system (Invitrogen; Thermo Fisher Scientific, Inc.). Briefly, recombination was produced by co-transfection of 293T cells with the lentivirus plasmid (pLV- PDHA1) and packaging plasmids (pLV1.0, pLV2.0 and pLV3.0) using the Lipofectamine 2000 transfection reagent (Invitrogen; Thermo Fisher Scientific, Inc.) according to the manufacturer's protocol.

Cell proliferation assay. Cell proliferation was assessed using the MTT assay. MTT was diluted in phosphate-buffered saline (PBS) to a final concentration of $5 \mathrm{mg} / \mathrm{ml}$ and sterile filtered. Cells were incubated with a final concentration of $0.5 \mathrm{mg} / \mathrm{ml}$ MTT at $37^{\circ} \mathrm{C}$ for $4 \mathrm{~h}$. Cell culture supernatants were carefully removed, and dimethyl sulfoxide (DMSO) was added. The absorbance values were determined using a microplate reader (Pharmacia Biotech, Uppsala, Sweden) at a wavelength of $570 \mathrm{~nm}$. The experiments were performed 3 times.

Colony formation assay. The cells were transfected with PDHA siRNA or control siRNA and seeded into a 6-well plate. Following 2 weeks of culture, the cells were fixed in methanol, stained with $0.1 \%(\mathrm{w} / \mathrm{v})$ crystal violet and washed with water. The cell colonies were scored and the representative light images were photographed using a Canon camera.

Glucose and lactate assessments. Glucose and lactate contents in culture medium were evaluated using the BS-200 Chemistry Analyzer (Mindray Bio-Medical Electronics Co., Ltd., Shenzhen, China) and EnzyChrom ${ }^{\mathrm{TM}}$ D-Lactate Assay kit (BioAssay Systems, Hayward, CA, USA), respectively. Data were normalized to the cell number in each well. For glucose uptake assays, the cells were maintained under normal conditions for $24 \mathrm{~h}$ and $10 \mu \mathrm{M}$ 2-NBDG (Life Technologies; Thermo Fisher Scientific, Inc.) was added to the medium for $30 \mathrm{~min}$ in the dark at $37^{\circ} \mathrm{C}$. After being washed with PBS 

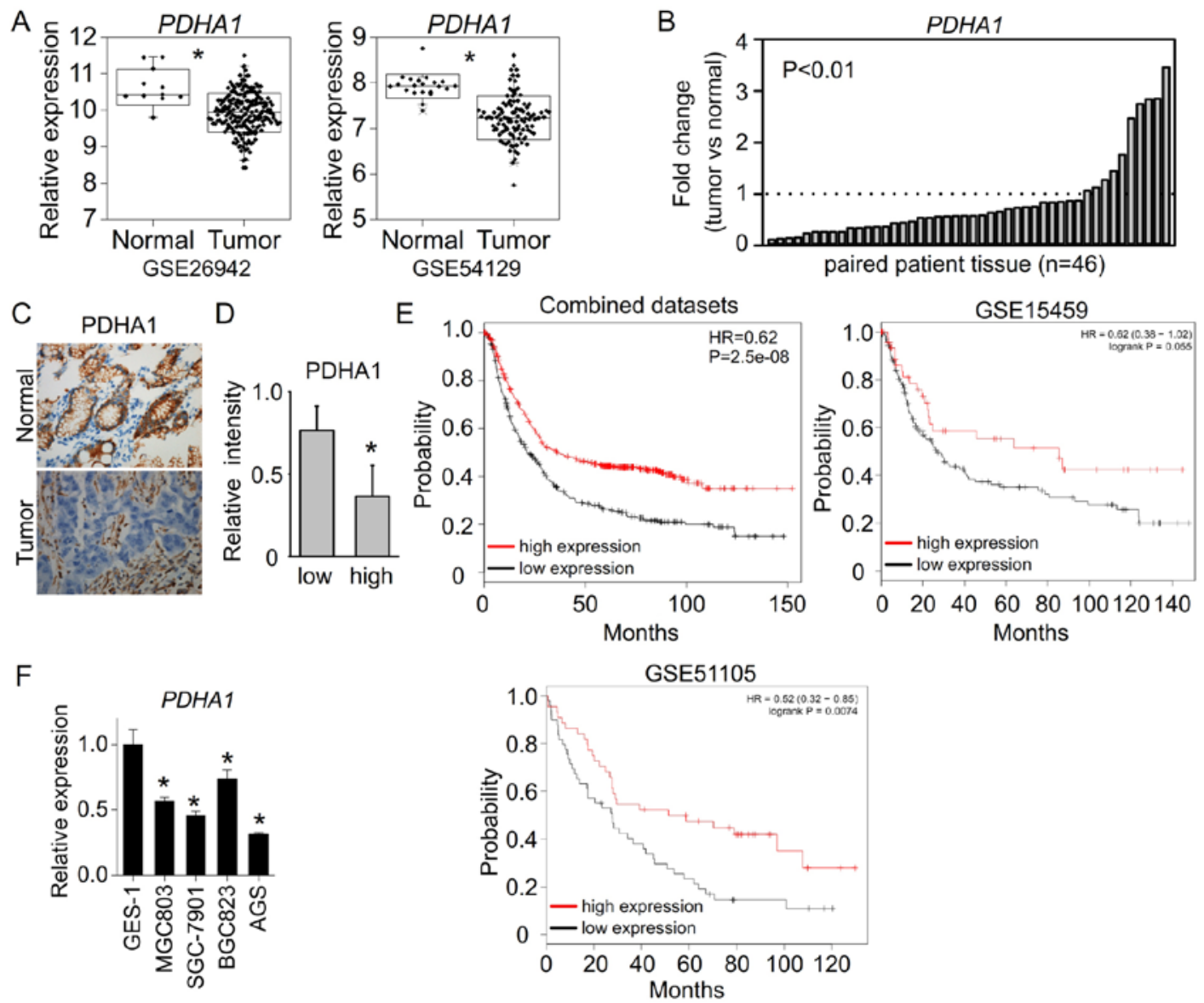

Figure 1.PDHA1 is downregulated in gastric cancer. (A) PDHA1 expression levels were analyzed in gastric cancer samples from patient datasets (GSE26942 and GSE54129). (B) PDHA1 was downregulated in gastric cancer tissues compared to adjacent non-tumor tissues, as examined by q-PCR. (C) PDHA1 was downregulated in gastric cancer tissues compared to adjacent non-tumor tissues, as examined by immunohistochemistry. (D) PDHA1 protein levels were downregulated in high-grade gastric cancer tissues. (E) PDHA1 expression levels were associated with poor survival. The analysis was done by online program Kaplan-Meier Plotter (http://kmplot.com/analysis/index.php?p=service\&cancer=gastric). Combined datasets include GSE14210, GSE15459, GSE22377, GSE29272, GSE51105 and GSE62254. (F) PDHA1 mRNA levels were examined in different cell lines. "P<0.05 compared to GES-1 cells. PDHA1, pyruvate dehydrogenase A1.

twice, the labeled cells were collected as single cell suspensions and the fluorescence intensities were determined by flow cytometry.

PDHA1 targeting miRNAs prediction. The putative miRNAs targeting PDHA1 were analyzed using online programs including DIANA TOOLS (http://diana.imis.athena-innovation. gr/DianaTools/index.php?r=microT_CDS/index), TargetScan 7.2 (http://www.targetscan.org/vert_72/) and miRDB (http://mirdb. org/miRDB/index.html).

Dual-Luciferase Reporter Assay. The 3'untranslated region (3'UTR) of PDHA1 containing the miR-21-5p binding sites was amplified by PCR from human genomic DNA. The wild-type 3'UTR of PDHA1 as well as the mutant 3'UTR with nucleotide substitutions in the putative binding sites corresponding to the seed sequence of miR-21-5p were inserted into the psiCHECK-2 vector immediately downstream of the stop codon of luciferase to develop psiCHECK2-PDHA1-3'UTR and psiCHECK-PDHA1-mut-3'UTR, respectively. Either of these vectors was co-transfected with miRNAs into cells using Lipofectamine 2000 according to the manufacturer's protocols. Luciferase activity was assessed $48 \mathrm{~h}$ after transfection using a Dual-Luciferase Reporter Assay kit (Promega Corp.,
Madison, WI, USA) and Victor Luminometer (PerkinElmer, Inc., Waltham, MA, USA). The firefly luciferase activity was normalized using co-transfected Renilla luciferase for transfection efficiency. Three independent experiments were performed and the data are presented as the mean \pm standard deviation (SD).

Protein extraction and western blot analysis. Total protein from cells was extracted with RIPA Lysis Buffer (Cell Signaling Technology, Inc.) and total protein was assessed using the Bradford protein assay (Bio-Rad Laboratories, Inc., Hercules, CA, USA). Protein (30 mg) were separated by $10 \%$ SDS-PAGE and transferred onto polyvinylidene fluoride (PVDF) membranes (EMD Millipore, Billerica, MA, USA). After blocking for non-specific binding with 5\% skimmed milk dissolved in Tris-buffered saline plus Tween-20 (TBS-T; $0.1 \%$ Tween-20; $\mathrm{pH} 8.3$ ) at room temperature for $1 \mathrm{~h}$, the membranes were incubated with antibodies against PDHA1 (1:1,000 dilution; cat. no. 3205) and $\beta$-actin (1:2,000 dilution; cat. no. 4970) (both from Cell Signaling Technology, Inc.) at $4^{\circ} \mathrm{C}$ overnight. After incubation with a secondary antibody conjugated to horseradish peroxidase (cat. no. 7074; Cell Signaling Technology) at room temperature for $1 \mathrm{~h}$, the protein bands were visualized using an enhanced chemiluminescence 

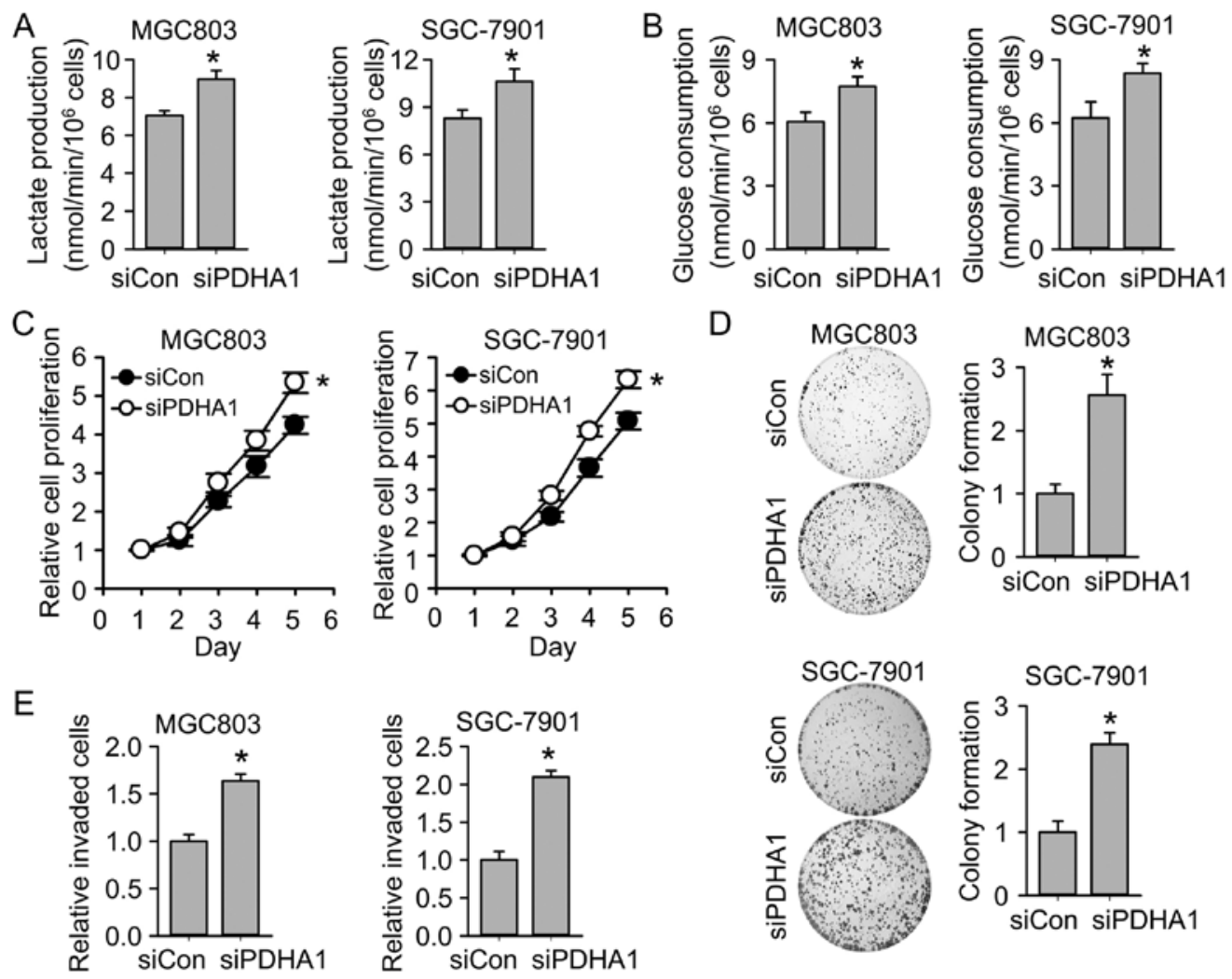

$\mathrm{F}$
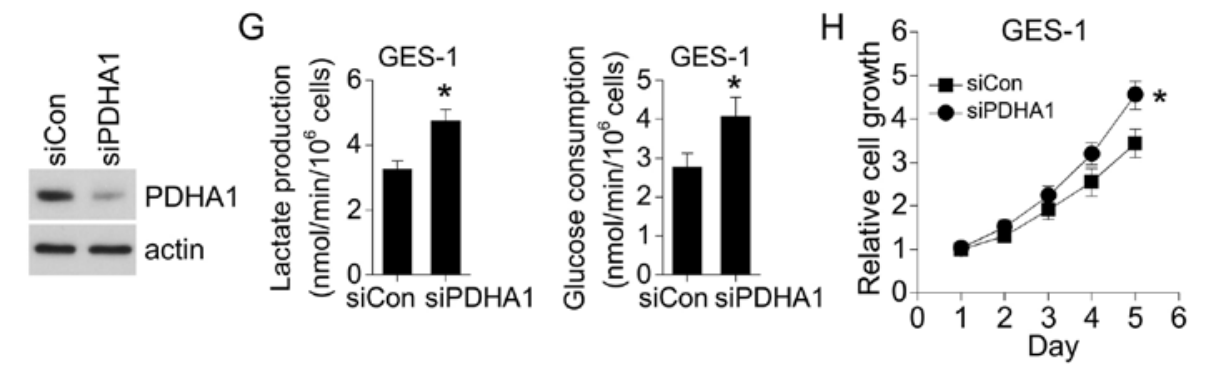

Figure 2. PDHA1 downregulation promotes glycolysis and cell proliferation. (A) Lactate production was assessed in cells at $72 \mathrm{~h}$ after PDHA1 knockdown. ${ }^{*} \mathrm{P}<0.05$. (B) Glucose consumption was assessed in cells at $72 \mathrm{~h}$ after PDHA1 knockdown. " $\mathrm{P}<0.05$. (C) Cell proliferation was assessed after PDHA1 knockdown. " $\mathrm{P}<0.05$. (D) Colony formation was assessed after PDHA1 knockdown. ${ }^{*} \mathrm{P}<0.05$. (E) Cell invasion was assessed after PDHA1 knockdown. "P<0.05. (F) PDHA1 was knocked down in GES-1 cells. (G) Lactate production and glucose consumption were assessed in PDHA1-knockdown GES-1 cells. "P $<0.05$. (H) Cell growth was assessed in PDHA1-knockdown GES-1 cells. "P<0.05. PDHA1, pyruvate dehydrogenase A1.

detection kit (EMD Millipore). ImageJ-1.51k software (developed by National Institutes of Health, Bethesda, MD, USA) was used for density measurement.

Statistical analysis. Data were analyzed using SPSS software 15.0 (SPSS, Inc., Chicago, IL, USA). Differences of PDHA1 and miR-21-5p expression between tumor tissues and adjacent non-tumor tissues were analyzed by the Wilcoxon matched-pairs test. The relationship between the expression of PDHA1 or miR-21-5p and the clinicopathologic grade was assessed by the $\chi^{2}$ test. Quantitative data are presented as the mean \pm the standard deviation. Statistical differences between groups were determined using the Student's t-test. Differences were considered significant when $\mathrm{P}<0.05$.

\section{Results}

PDHAl is downregulated in human gastric cancer. Pyruvate dehydrogenase $(\mathrm{PDH})$ is a multienzyme complex that functions as a gatekeeper in glucose metabolism by oxidatively decarboxylating pyruvate to produce acetyl-CoA for the TCA cycle, and therefore is closely related to increased glycolysis in cancer. PDHA1 is the main component of PDH, which catalyzes pyruvate decarboxylation and serves as a gate-keeper enzyme link between glycolysis and the mitochondrial citric acid cycle. To determine the role of PDHA1 in gastric cancer, we determined whether PDHA1 is dysregulated in gastric cancer patient samples. By analyzing the gastric cancer patient datasets, we found that PDHA1 expression was significantly downregulated in gastric cancer patient samples compared to normal gastric tissues (GSE26942 and GSE54120) (Fig. 1A). In our paired gastric cancer samples, the mRNA levels of PDHA1 were also downregulated in 36 out of 46 gastric cancer tissues compared to their adjacent non-tumor tissues (Fig. 1B). Moreover, we further assessed the protein levels of PDHA1 in gastric cancer tissues. The similar results revealed that PDHA1 protein levels were also downregulated in gastric cancer tissues compared to their adjacent normal tissues (Fig. 1C). In 

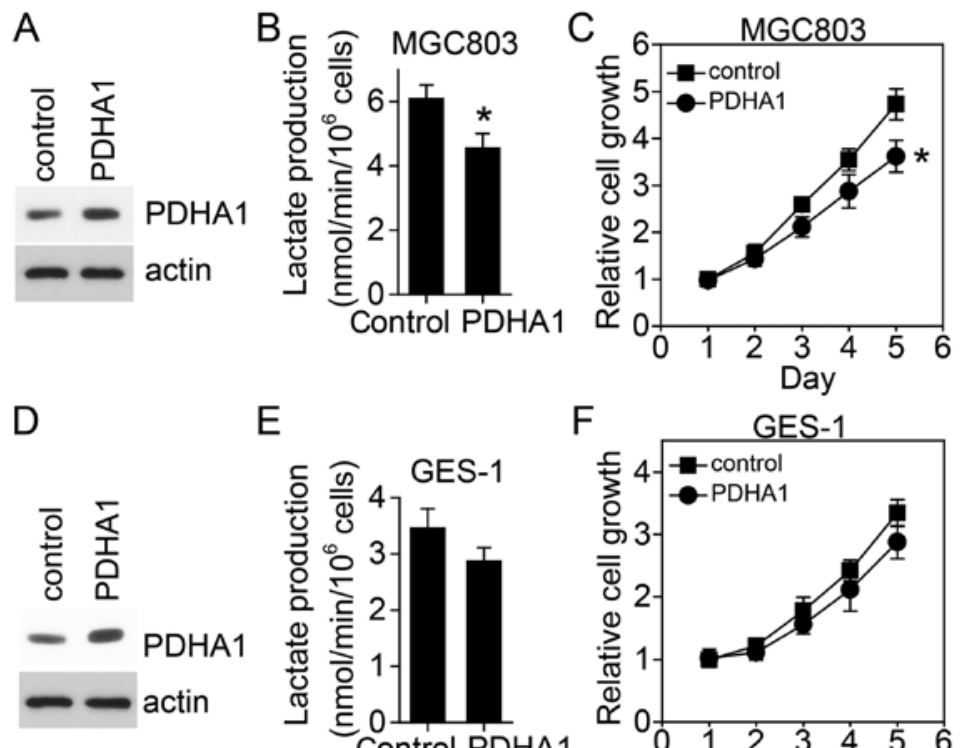

$\mathrm{E}$

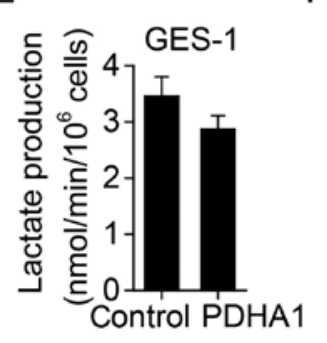

$\mathrm{F}$

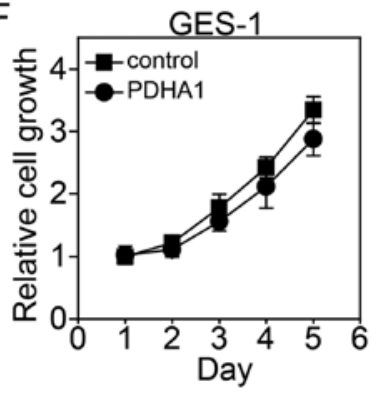

Figure 3. PDHA1 overexpression affects glycolysis and cell growth of gastric cancer cells. (A) PDHA1 was overexpressed in MGC803 cells. (B) Lactate production was assessed in PDHA1-overexpressed MGC803 cells. " $\mathrm{P}<0.05$. (C) Cell growth was assessed in PDHA1-overexpressed MGC803 cells. " $\mathrm{P}<0.05$. (D) PDHA1 was overexpressed in GSE-1 cells. (E) Lactate production was assessed in PDHA1-overexpressed GSE-1 cells. (F) Cell growth was assessed in PDHA1-overexpressed GSE-1 cells. PDHA1, pyruvate dehydrogenase A1.

addition, the PDHA1 levels were less expressed in high grade gastric cancer tissues (Fig. 1D), suggesting that the downregulation of PDHA1 may be correlated with gastric cancer progression. The survival analysis of patients further revealed that low PDHA1 was associated with poor survival (Fig. 1E). We further assessed the expression levels of PDHAlin gastric cancer cell lines. The results revealed that PDHA1 expression was significantly reduced in gastric cancer cell lines compared to normal gastric cell line GES-1 (Fig. 1F). These data indicated that PDHA1 was downregulated in gastric cancer and involved in gastric cancer development.

PDHA1 downregulation promotes glycolysis and cancer progression. To examine the role of PDHA1 downregulation in gastric cancer progression, we knocked down PDHA1 in gastric cancer cell lines MGC803 and SGC-7901. Consistent with the role of PDHA1 in regulating glycolysis, our results revealed that knockdown of PDHA1 promoted glycolysis. Lactate production and glucose consumption were significantly increased following PDHA1 knockdown (Fig. 2A and B). Moreover, we found that PDHA1 knockdown significantly promoted cell proliferation (Fig. 2C). Consistently, the colony formation ability of gastric cancer cells was also enhanced after PDHA1 knockdown (Fig. 2D). Our results also revealed that PDHA1 knockdown promoted gastric cancer invasion (Fig. 2E). All these results indicated that PDHA1 downregulation in gastric cancer cell enhanced glycolysis and promoted gastric cancer progression. Notably, we found that knockdown of PDHA1 in normal gastric cells (Fig. 2F) also resulted in increased glycolysis and cell growth (Fig. 2G and $\mathrm{H}$ ), further supporting that PDHA1 downregulation plays a tumor promoting role. In contrast, overexpression of PDHA1 in gastric cancer cells reduced glycolysis and resulted in cell growth inhibition (Fig. 3A-C). Even in normal gastric cells, overexpression of PDHA1 also inhibited glycolysis and cell growth, however, not significantly (Fig. 3D-F).
miR-21-5p targets PDHAl in gastric cancer cells. miRNAs target transcript mRNAs to regulate gene expression and play important roles in cancer. To investigate the miRNA which regulates PDHA1 in gastric cancer, we searched putative miRNAs targeting PDHA1 using online programs including DIANA, TargetScan and miRDB. DIANA revealed 82 candidate miRNAs targeting PDHA1, TargetScan revealed 108 candidate miRNAs, and miRDB revealed 74 candidate miRNAs. By comparing the common miRNAs, we found 29 miRNAs targeting PDHA1 predicted by all these three programs (Fig. 4A), suggesting they likely target PDHA1. To further investigate which one might target PDHA1 in gastric cancer, we first compared their expression in clinical gastric cancer samples. Several of these candidates exhibited dysregulation in gastric cancer samples compared to normal gastric samples. Among them, miR-21-5p exhibited the most significant upregulation in gastric cancer samples (Fig. 4B), which was consistent with PDHA1 downregulation in gastric cancer. We further examined whether miR-21-5p targets PDHA1 in gastric cancer cells. miR-21-5p mimics were transfected into MGC-803 cells, and the mRNA and protein levels of PDHA1 were examined. The results revealed that miR-21-5p transfection significantly reduced PDHA1 mRNA and protein levels in gastric cancer cells (Fig. 4C and D). Consistently, miR-21-5p had a conserved binding site on the 3'UTR of PDHA1 mRNA (Fig. 4E). We further used a luciferase assay to confirm PDHA1 as a target of miR-21-5p. The results revealed that miR-21-5p reduced the PDHA1 3'UTR-derived luciferase activity, but did not affect luciferase activity derived by PDHA1 3'UTR with the mutant miR-21-5p binding site (Fig. 4F). These data indicated that miR-21-5p directly targeted PDHA1 mRNA to suppress PDHA1 expression.

miR-21-5p is upregulated in gastric cancer. We further confirmed miR-21-5p upregulation in our gastric cancer 


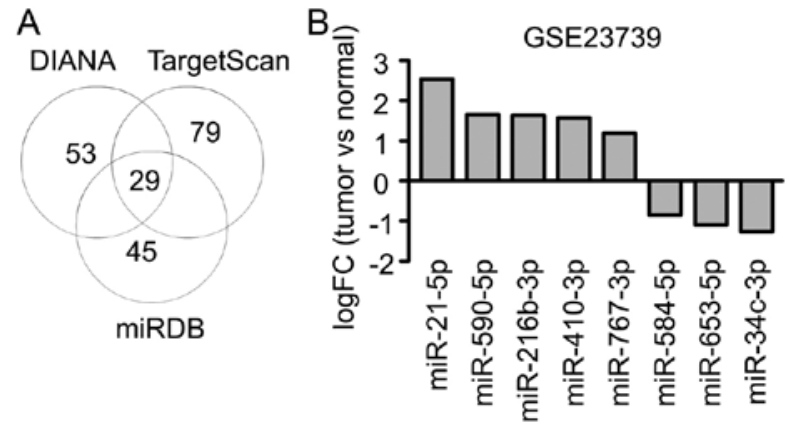

E
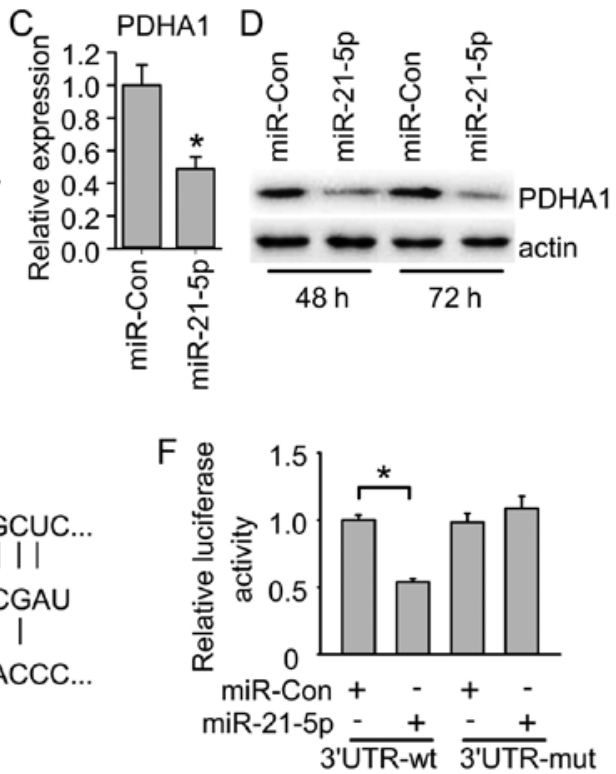

Figure 4. miR-21-5p targets PDHA1. (A) Predicted miRNAs by DIANA, TargetScan and miRDB were compared. (B) The candidate miRNAs were dysregulated in gastric cancer patient samples (GSE23739). (C) PDHA1 mRNA levels were reduced by miR-21-5p. "P<0.05. (D) PDHA1 protein levels were reduced by miR-21-5p. (E) Schema of binding site of miR-21-5p in the PDHA1 3'UTR region. (F) PDHA1 3'UTR-driven luciferase activity was reduced by miR-21-5p. ${ }^{*} \mathrm{P}<0.05$. PDHA1, pyruvate dehydrogenase A1.
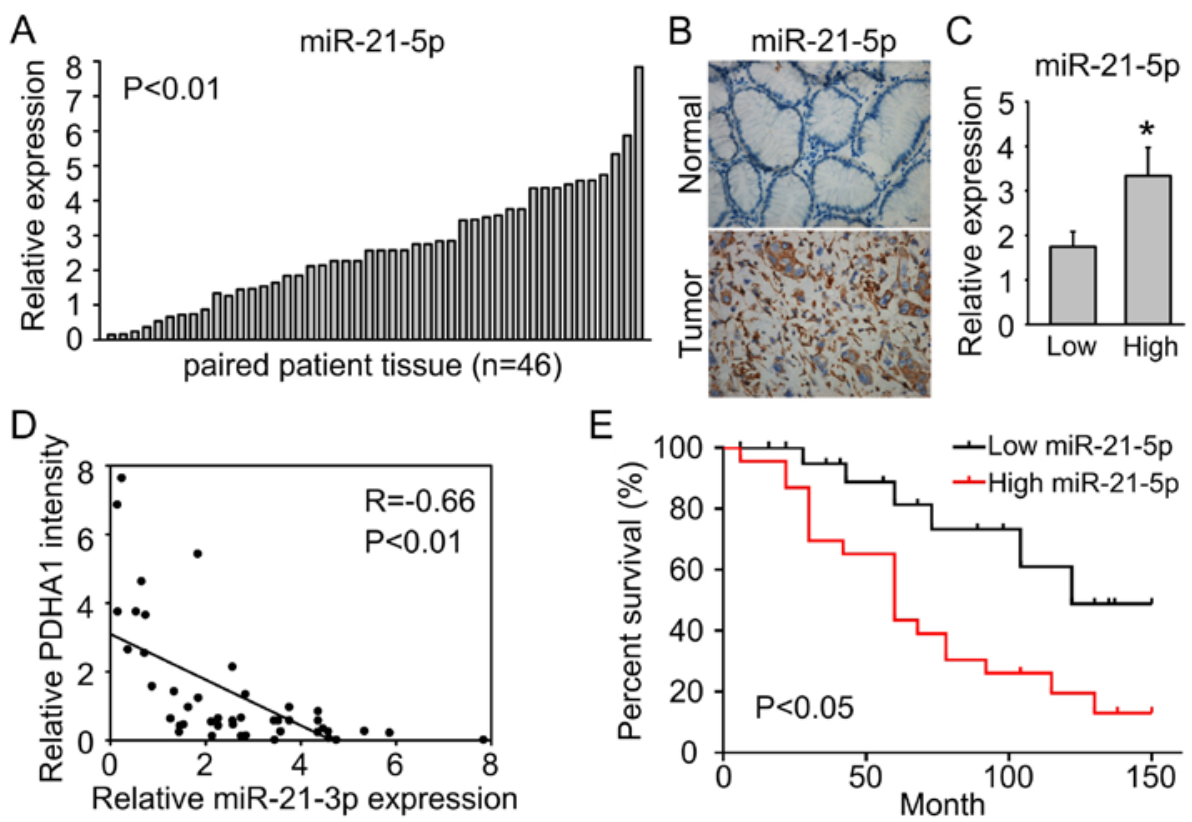

Figure 5. miR-21-5p is upregulated in gastric cancer and negatively associated with PDHA1. (A) miR-21-5p was upregulated in gastric cancer tissues compared to adjacent non-tumor tissues, as examined by q-PCR. (B) miR-21-5p was upregulated in gastric cancer as examined by in situ hybridization. (C) miR-21-5p was correlated with tumor grade. ${ }^{*} \mathrm{P}<0.05$. (D) miR-21-5p was negatively associated with PDHA1 expression. (E) High miR-21-5p expression levels were associated with poor survival. PDHA1, pyruvate dehydrogenase A1.

samples. We assessed the expression levels of miR-21-5p in 46 pairs of gastric cancer tissue samples by q-PCR analysis. As shown in Fig. 5A, miR-21-5p was significantly upregulated in gastric cancer tissues compared to adjacent non-tumor tissues $(\mathrm{P}<0.01)$. The results of in situ hybridization analysis also revealed that miR-21-5p was upregulated in gastric cancer tissues (Fig. 5B). Notably, miR-21-5p was significantly upregulated in high grade gastric cancer compared to low grade tissues (Fig. 5C). Moreover, PDHA1 was negatively associated with miR-21-5p in gastric cancer samples (Fig. 5D), further supporting that miR-21-5p targets PDHA1 in gastric cancer. In addition, high miR-21-5p expression was associated with poor survival (Fig. 5E).

miR-21-5p regulates glycolysis and inhibits gastric cancer cell growth. Since miR-21-5p targeted PDHA1 in gastric cancer, we next examined whether miR-21-5p also regulated glycolysis and cell growth. We adjusted miR-21-5p expression through transfection of miR-21-5p mimics and its inhibitor (Fig. 6A). The results revealed that transfection of miR-21-5p mimics 

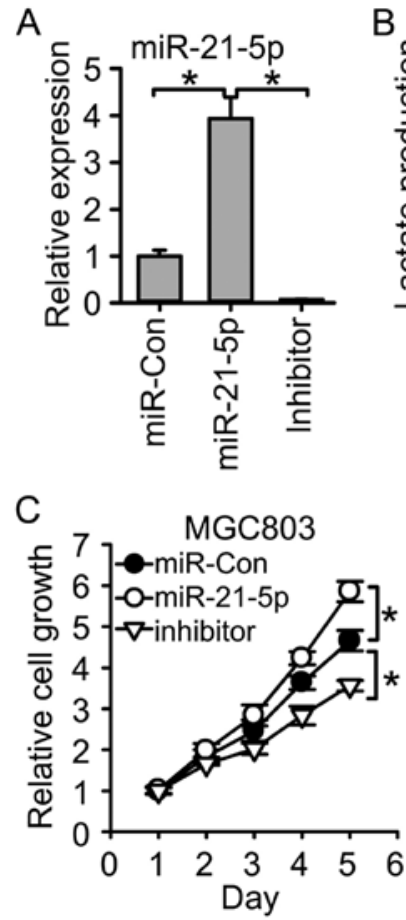
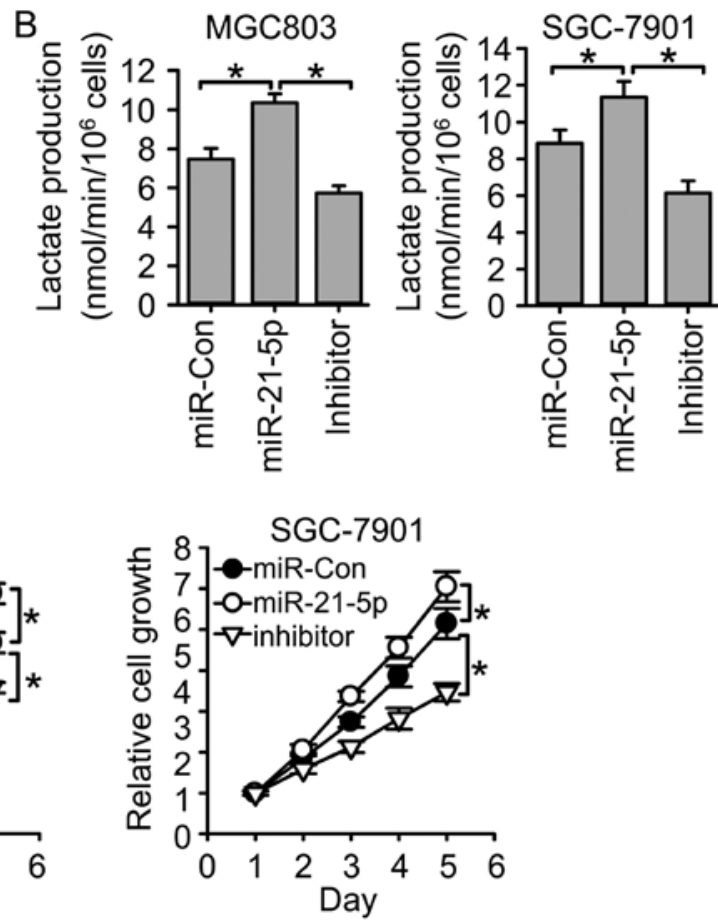

Figure 6. miR-21-5p promotes gastric cancer cell proliferation. (A) miR-21-5p expression was assessed after transfection of miR-21-5p or its inhibitor. "P<0.05. (B) Lactate production was assessed $72 \mathrm{~h}$ after transfection of miR-21-5p or its inhibitor. ${ }^{*} \mathrm{P}<0.05$. (C) Cell growth was assessed $72 \mathrm{~h}$ after transfection of miR-21-5p or its inhibitor. ${ }^{*} \mathrm{P}<0.05$.
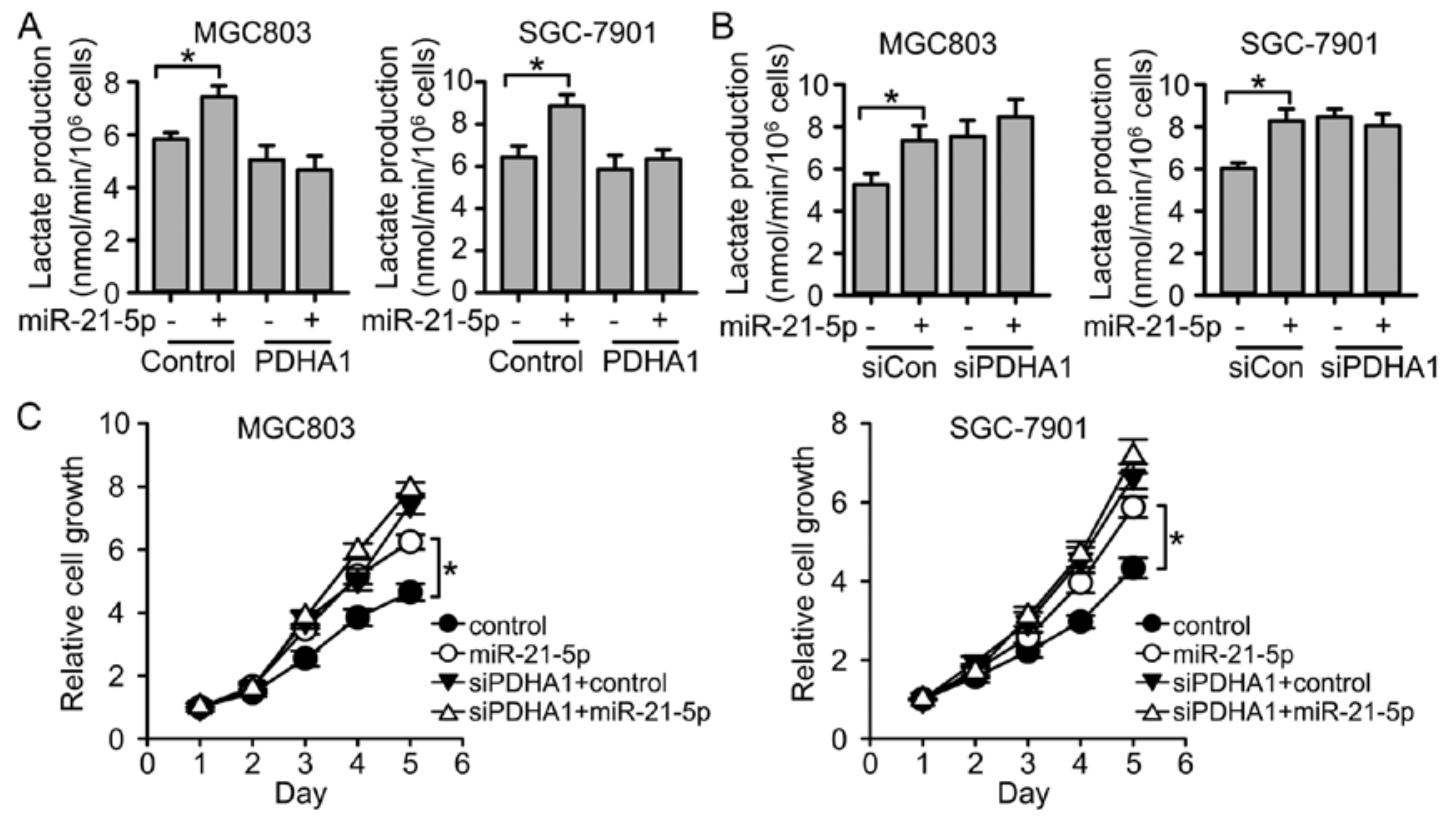

Figure 7. miR-21-5p targets PDHA1 to regulate cell proliferation. (A) Lactate production was assessed $72 \mathrm{~h}$ after transfection of miR-21-5p in PDHA1-overexpressed cells. ${ }^{*} \mathrm{P}<0.05$. (B) Lactate production was assessed $72 \mathrm{~h}$ after transfection of miR-21-5p and/or siRNA targeting PDHA1. "P<0.05. (C) Cell proliferation was assessed after transfection of miR-21-5p and/or siRNA targeting PDHA1. "P<0.05. PDHA1, pyruvate dehydrogenase A1.

increased lactate production, while transfection of miR-21-5p inhibitor reduced lactate production (Fig. 6B), suggesting that miR-21-5p promotes glycolysis in gastric cancer. In addition, miR-21-5p overexpression promoted gastric cancer cell growth, while miR-21-5p inhibition reduced gastric cancer cell growth (Fig. 6C). These results indicated the tumor promoting role in gastric cancer.
miR-21-5p targets PDHA1 to regulate glycolysis and gastric cancer cell growth. Next, we investigated whether miR-21-5p regulated glycolysis and cell growth through PDHA1. miR-21-5p and PDHA1 were both overexpressed in gastric cancer cell lines. We revealed that miR-21-5p promoted glycolysis in control cells, but failed to promote glycolysis in PDHA1-overexpressed cells (Fig. 7A). Consistently, when we 
suppressed PDHA1 expression, miR-21-5p failed to further increase the levels of glycolysis (Fig. 7B). The results indicated that miR-21-5p regulated glycolysis through PDHA1. Moreover, we found that miR-21-5p promoted cell proliferation, while PDHA1 knockdown impaired the effect of miR-21-5p on cell proliferation (Fig. 7C). All the results indicated that miR-21-5p targets PDHA1 to regulate glycolysis and gastric cancer cell proliferation.

Collectively, we revealed that PDHA1 was downregulated in gastric cancer, and PDHA1 downregulation promoted glycolysis and cancer progression. miR-21-5p upregulation contributed to PDHA1 downregulation and regulated gastric cancer cell progression. Our study indicated that miR-21-5p and PDHA1 are involved in gastric cancer progression, and suggests that we could target miR-21-5p to modulate PDHA1 expression and benefit gastric cancer treatment.

\section{Discussion}

In the present study, we demonstrated that PDHA1 was downregulated in gastric cancer cells, and PDHA1 downregulation promoted glycolysis and resulted in enhanced cell proliferation, colony formation and invasion of gastric cancer cells. Further study revealed that miR-21-5p was upregulated in gastric cancer, and targeted PDHA1 to suppress PDHA1 expression. miR-21-5p functioned through PDHA1 to regulate glycolysis and gastric cancer progression. Our results indicated that the promotion of glycolysis in gastric cancer was dependent on the downregulation of PDHA1, resulting from the upregulation of miR-21-5p.

Cancer cells have a distinct metabolic pattern with enhanced glycolysis levels, that is known as the famous 'Warburg effect' $(4,5)$. This phenomenon is considered as one of the important hallmarks of cancer (3), and has been taken advantage to benefit cancer diagnosis and therapy. Clinically, high glucose uptake is used to diagnose or monitor the treatment responses of cancers by imaging the uptake of 2-18 F-deoxyglucose with PET-CT (3). Many metabolic enzymes have been found to serve as therapeutic targets for cancer treatment. Pyruvate metabolic enzymes regulate pyruvate transferring from the glycolytic pathway to the TCA cycle, and thus play essential roles in cancer cell metabolism and cancer progression. Pyruvate dehydrogenase (PDH) is a multienzyme complex consisting of multiple copies of E1, E2 and E3 subunits, along with an E3 binding protein (E3BP), which serves to bind E3 to the complex. PDH functions as a gatekeeper in glucose metabolism by oxidatively decarboxylating pyruvate to produce acetyl-CoA for the TCA cycle. Therefore, this enzyme plays an important role in the metabolic node (6). PDHA1 is the main component of PDH, which catalyzes pyruvate decarboxylation and serves as a gate-keeper enzyme link between glycolysis and the mitochondrial citric acid cycle. In the present study, we revealed that downregulation of PDHA1 in gastric cancer cells was associated with poor survival, and led to increased glycolysis and promoted cancer progression. These results were consistent with the phenotype caused by dysregulation of other pyruvate-associated enzymes such as pyruvate dehydrogenase kinase 1 (PDK1) and mitochondrial pyruvate carrier (MPC). Consistent with the tumor-suppressive role of PDHA1, we revealed that PDHA1 was downregulated in gastric cancer tissues, particularly in high-grade tumors.
Considering the tumor-suppressive role of PDHA1 in cancer, it is important to readjust PDHA1 expression to modulate cancer metabolism and cell growth. Thus, it is necessary to investigate how PDHA1 expression is regulated. A miRNA can regulate gene expression by binding to the 3'UTR of the target mRNA. miRNAs play important roles in most cellular processes by regulating the protein expression of target genes. It has been reported that miRNAs are dysregulated in various types of human cancers. The dysregulation of miRNAs leads to altered expression of target genes including tumor suppressors and oncogenes, and regulates tumor promotion and progression. To determine which miRNA regulates PDHA1 in gastric cancer, we first analyzed the common miRNAs targeting PDHA1 in three well known miRNA prediction programs, and then analyzed which miRNAs were upregulated in gastric cancer. These miRNAs were likely to target PDHA1 in gastric cancer. Among these candidates, miR-21-5p was the most highly upregulated miRNA in gastric cancer, and we further confirmed that it directly targeted PDHA1 expression. We demonstrated that overexpressed miR-21-5p significantly suppressed PDHA1 expression, and more importantly, miR-21-5p expression was negatively associated with PDHA1 expression in clinical gastric cancer samples.

miR-21 has been frequently reported to be aberrantly overexpressed in diverse tumors, including glioblastoma, breast cancer and malignant cholangiocytes $(22,23)$. Recently, miR-21-5p was also reported to be overexpressed in gastric cancer (24). Previous studies demonstrated that miR-21-5p plays a tumor-promoting role in many types of cancer. It functions by regulating several oncogenic pathways such as $\mathrm{PI} 3 \mathrm{~K} / \mathrm{AKT}$ and modulating matrix metalloproteases $(25,26)$. Consistently, we found that miR-21-5p was upregulated in gastric cancer tissues and miR-21-5p expression was associated with tumor grade, and exhibited high expression in high-grade tumors and low expression in low-grade tumors. Inhibition of miR-21-5p led to reduced cell growth. The results revealed that miR-21-5p serves as an oncogene in gastric cancer. However, the role of miR-21-5p in cancer metabolism has not been studied. We revealed for the first time that miR-21-5p overexpression promoted glycolysis and cell proliferation in gastric cancer cells; miR-21-5p targeted PDHA1 to regulate glycolysis and cancer progression. Either forced overexpression or knockdown of PDHA1 could abrogate miR-21-5p-induced glycolysis. This suggests that miR-21-5p regulated glycolysis in gastric cancer mainly through PDHA1.

In the present study, we demonstrated that PDHA1, the essential pyruvate metabolism enzyme, is downregulated in gastric cancer cells, and PDHA1 downregulation promoted glycolysis and cancer cell growth. We further revealed that PDHA1 was a direct target of miR-21-5p. miR-21-5p was upregulated in gastric cancer, and suppressed PDHA1 expression to promote glycolysis and cancer cell growth. In summary, our study illustrated that the miR-21-5p/PDHA1 axis was involved in gastric cancer glycolysis and progression, suggesting their potential benefit in gastric cancer treatment.

\section{Acknowledgements}

Not applicable. 


\section{Funding}

The present study was supported in part by a grant from the Jilin Programs for Science and Technology Development (no. 20160101034JC).

\section{Availability of data and materials}

The datasets used during the present study are available from the corresponding author upon reasonable request.

\section{Authors' contributions}

ZL, TM and DW conceived and designed the study. ZL, MY, BF and XF performed the experiments. ZL wrote the manuscript. ZL, XF, TM and DW reviewed and edited the manuscript. All authors read and approved the manuscript and agree to be accountable for all aspects of the research in ensuring that the accuracy or integrity of any part of the work are appropriately investigated and resolved.

\section{Ethics approval and consent to participate}

All experimental protocols were approved by the Ethics Committee of China-Japan Union Hospital of Jilin University. Written informed consent was obtained from all the patients or their guardians in accordance with the ethical committee standards.

\section{Patient consent for publication}

Not applicable.

\section{Competing interests}

The authors state that they have no competing interests.

\section{References}

1. Siegel R, Naishadham D and Jemal A: Cancer statistics, 2013. CA Cancer J Clin 63: 11-30, 2013.

2. Hamashima C, Shabana M, Okada K, Okamoto M and Osaki Y: Mortality reduction from gastric cancer by endoscopic and radiographic screening. Cancer Sci 106: 1744-1749, 2015.

3. Hanahan D and Weinberg RA: Hallmarks of cancer: The next generation. Cell 144: 646-674, 2011.

4. Warburg O: On the origin of cancer cells. Science 123: 309-314, 1956.

5. Warburg O: On respiratory impairment in cancer cells. Science 124: 269-270, 1956.

6. Bresters TW, de Kok A and Veeger C: The pyruvate-dehydrogenase complex from Azotobacter vinelandii. 2. Regulation of the activity. Eur J Biochem 59: 347-353, 1975.
7. Kim JW, Tchernyshyov I, Semenza GL and Dang CV: HIF-1-mediated expression of pyruvate dehydrogenase kinase: A metabolic switch required for cellular adaptation to hypoxia. Cell Metab 3: 177-185, 2006.

8. Dupuy F, Tabaries S, Andrzejewski S, Dong Z, Blagih J, Annis MG, Omeroglu A, Gao D, Leung S, Amir E, et al: PDK1-dependent metabolic reprogramming dictates metastatic potential in breast cancer. Cell Metab 22: 577-589, 2015.

9. Li Y, Li X, Zhong Y, Ji Y, Yu D, Zhang M, Wen JG, Zhang H, Goscinski MA, Nesland JM, et al: PDHAl gene knockout in prostate cancer cells results in metabolic reprogramming towards greater glutamine dependence. Oncotarget 7: 53837-53852, 2016.

10. Liu F, Zhang W, You X, Liu Y, Li Y, Wang Z, Wang Y, Zhang X and Ye L: The oncoprotein HBXIP promotes glucose metabolism reprogramming via downregulating $\mathrm{SCO} 2$ and PDHA1 in breast cancer. Oncotarget 6: 27199-27213, 2015.

11. Li Y, Huang R, Li X, Li X, Yu D, Zhang M, Wen J, Goscinski MA, Trope CG, Nesland JM, et al: Decreased expression of pyruvate dehydrogenase A1 predicts an unfavorable prognosis in ovarian carcinoma. Am J Cancer Res 6: 2076-2087, 2016.

12. Lin CS, Lee HT, Lee MH, Pan SC, Ke CY, Chiu AW and Wei YH: Role of mitochondrial DNA copy number alteration in human renal cell carcinoma. Int J Mol Sci 17: E814, 2016.

13. Filipowicz W, Bhattacharyya SN and Sonenberg N: Mechanisms of post-transcriptional regulation by microRNAs: Are the answers in sight? Nat Rev Genet 9: 102-114, 2008.

14. Hao NB, He YF, Li XQ, Wang K and Wang RL: The role of miRNA and lncRNA in gastric cancer. Oncotarget 8: 81572-81582, 2017.

15. Bartel DP: MicroRNAs: Genomics, biogenesis, mechanism, and function. Cell 116: 281-297, 2004.

16. Bartels CL and Tsongalis GJ: MicroRNAs: Novel biomarkers for human cancer. Clin Chem 55: 623-631, 2009.

17. Lai WF and Siu PM: MicroRNAs as regulators of cutaneous wound healing. J Biosci 39: 519-524, 2014.

18. Ambros V: The functions of animal microR NAs. Nature 431: 350-355, 2004.

19. Farh KK, Grimson A, Jan C, Lewis BP, Johnston WK, Lim LP, Burge CB and Bartel DP: The widespread impact of mammalian MicroRNAs on mRNA repression and evolution. Science 310: 1817-1821, 2005.

20. Yi R, O'Carroll D, Pasolli HA, Zhang Z, Dietrich FS, Tarakhovsky A and Fuchs E: Morphogenesis in skin is governed by discrete sets of differentially expressed microRNAs. Nat Genet 38: 356-362, 2006.

21. Kloosterman WP and Plasterk RH: The diverse functions of microRNAs in animal development and disease. Dev Cell 11: 441-450, 2006.

22. Masoudi MS, Mehrabian E and Mirzaei H: MiR-21: A key player in glioblastoma pathogenesis. J Cell Biochem 119: 1285-1290, 2018.

23. Han JG, Jiang YD, Zhang CH, Yang YM, Pang D, Song YN and Zhang GQ: A novel panel of serum miR-21/miR-155/miR-365 as a potential diagnostic biomarker for breast cancer. Ann Surg Treat Res 92: 55-66, 2017.

24. Sekar D, Krishnan R, Thirugnanasambantham K, Rajasekaran B, Islam VI and Sekar P: Significance of microRNA 21 in gastric cancer. Clin Res Hepatol Gastroenterol 40: 538-545, 2016.

25. Wang P, Guan Q, Zhou D, Yu Z, Song Y and Qiu W: miR-21 inhibitors modulate biological functions of gastric cancer cells via PTEN/PI3K/mTOR Pathway. DNA Cell Biol 37: 38-45, 2018.

26. Shen KH, Hung JH, Chang CW, Weng YT, Wu MJ and Chen PS: Solasodine inhibits invasion of human lung cancer cell through downregulation of miR-21 and MMPs expression. Chem Biol Interact 268: 129-135,2017. 